\title{
THE NARDO-GNAPHALIETUM SUPINI PLANT ASSOCIATION IN THE WESTERN CARPATHIANS (THE TATRA MTS AND THE BABIA GÓRA MASSIF)
}

\author{
PIOTR GÓRSKI \\ Department of Botany, August Cieszkowski Agricultural University \\ Wojska Polskiego 71C, 60-625 Poznań, Poland \\ e-mail: peter@owl.au.poznan.pl
}

(Received: January 15, 2003. Accepted: December 12, 2003)

\begin{abstract}
This article is the first published information on the occurrence of the snow-beds of Nardo-Gnaphalietum supini in the Western Carpathians. So far, localities of Nardo-Gnaphalietum supini phytocoenoses have been known exclusively from the Alps, the Black Forest, and the Eastern Carpathians. During this study, carried out in the Polish Carpathians, phytocoenoses of Nardo-Gnaphalietum were recorded in the two highest massifs - the Tatra Mountains and the Babia Góra Massif. They were closely related to anthropogenically-influenced sites with exposed and lumped ground located near tourist routes. Habitat characterization and spatial complexes of the patches of Nardo-Gnaphalietum in the investigated area are given herein.

Critical revision of phytosociological data from the Alps and the Eastern Carpathians revealed that the discussed phytocoenoses have been described within the communities of Luzuletum alpino-pilosae, Salicetum herbaceae or Nardetum strictae. In this study, all phytosociological data concerning the association were collected and the floristic distinction of the phytocoenoses of particular mountain massifs is discussed. A new subassociation Nardo-Gnaphalietum oligotrichetosum hercynici was distinguished on the basis of local habitat variability. Although Nardo-Gnaphalietum supini does not have characteristic species, it is a well-distinguished typological unit in the class Salicetea herbaceae Br.-B1. 1948, and is a central association of the alliance Nardo-Salicion herbaceae Englisch 1999.
\end{abstract}

KEY WORDS: Nardo-Gnaphalietum supini, Salicetea hebraceae, snow-bed vegetation, Tatra Mts, Babia Góra Massif, Western Carpathians.

\section{INTRODUCTION}

Phytocoenoses of Nardo-Gnaphalietum supini have not been, as yet, known from the Western Carpathians. All available information on their occurrence refers to the Alps, the Black Forest and the Eastern Carpathians. This syntaxon has been first documented over sixty years ago (Bartsch J. and Bartsch M. 1940) with a single phytosociological relevé and described as 'Nardus-Gnaphalium supinum-Gesellschaft' from the Black Forest Mountains. That patch overgrowed a hollow within the dense Vaccinietum myrtilli community in the subalpine zone of the Feldberg Massif. However another relevé made in the same area, was regarded as representing 'Nardus stricta-Gnaphalium supinum-Association' (Müller 1948). Oberdorfer (1950), although not questioning the distinctiveness of the NardusGnaphalium community, considered it as merely a variant (with Nardus stricta) of the association Luzuletum alpinopilosae Br.-Bl. in Br.-Bl. et Jenny 1926 (see Table 16, rel.
1 in the cited paper). Materials by Thimm (1953, included in the table of Salicetum herbaceae Br.-B1. 1913, variant with Mutellina purpurea cf. Table 25, col. 3, 4 and 5) may also be listed as belonging to Nardo-Gnaphalietum. The mentioned variant of the association distinguishes itself negatively by the lack of Salix herbacea. Other data may be found in studies by Bolleter (1921), under the name of Nardetum with Plantago alpina, and by Eggensberger (1994). Another documentation from the Eastern Carpathians consists of 8 relevés from the Rodney Mountains (Coldea 1990) and other 8 records from the Retezat (Resmerita 1975; the relevés included in tables of Nardetum strictae alpinum and N. s. a. poetosum mediae).

As may be derived from the above review, the documentation referring to Nardo-Gnaphalietum supini is still insufficient and scattered in various phytosociological tables under different names. The aim of this study was to provide a scientific description of the Western Carpathians' phytocoenoses of Nardo-Gnaphalietum supini and to di- 
scuss the relations of their floristic composition to the published material from the whole distribution area of the association.

\section{MATERIALS AND METHODS}

The original material consisted of 16 phytosociological relevés made by using the classic Braun-Blanquet's (1951) method (cf. Dierschke 1994). The modified quantitative scale (2a, 2b, 2m) of Barkman et al. (1964) was implemented for assessment of the species' cover. The constancy values and cover coefficients were computed according to the procedure proposed by Pawłowski (1966). The names of vascular plants are in accordance with the list of Mirek et al. (1995), Ochyra et al. (1992) for mosses, and Grolle (1983) for liverworts. Altogether, 27 published phytosociological relevés were taken into consideration in comparative tables.

The investigations were carried out in 1995-1999 in all massifs of high mountain character in the Polish part of the Carpathians. The patches of Nardo-Gnaphalietum supini were recorded from the Tatra Mountains and the Babia Góra Massif. The first one is the highest mountain range in the whole Carpathians (with Gerlach elevated at 2663 $\mathrm{m}$ a.s.1.). It is a borderline mountain chain situated between Poland and Slovakia, with the greater part belonging to the latter country. The Babia Góra (Diablak, $1725 \mathrm{~m}$ a.s.1.) is the second highest mountain range in Poland. It is the culmination of the Beskid Wysoki in the Western Carpathians.

\section{RESULTS}

Patches of Nardo-Gnaphalietum supini were recorded in non-calcareous parts of the Tatra Mountains and in the Ba- bia Góra Massif (Table 1). They have established in both forest belts and the subalpine belt, and were observed along tourist routes parallel to contour lines (on the upper side of the slope above the route) and on non-marked footpaths within the subalpine communities of bilberry and mat grass communities.

Phytocoenoses of Nardo-Gnaphalietum supini occupy distinctly marked places in the mountain vegetation landscape. In areas potentially covered by montane spruce forests they occur in a spatial complex with: Plagiothecio-Piceetum (Szaf., Pawł. et Kulcz. 1932) Br.-Bl., Vlieg. et Siss. 1939 em. J. Mat. 1978 (P-P), Hieracio-Nardetum Kornaś 1955 (H-N), and Vaccinietum myrtilli Szaf., Pawł. et Kulcz. $1923(\mathrm{Vm})$. The mentioned vegetation appear in following linear patterns (Fig. 1):

forest edge $(\mathrm{P}-\mathrm{P}) \rightarrow \mathrm{Vm} \rightarrow \mathrm{H}-\mathrm{N} \rightarrow$ Nardo-Gnaphalietum $\rightarrow$ [tourist route] $\rightarrow$ forest edge (P-P)

or

forest edge $(\mathrm{P}-\mathrm{P}) \rightarrow \mathrm{H}-\mathrm{N} \rightarrow$ Nardo-Gnaphalietum $\rightarrow$ [tourist route] $\rightarrow$ forest edge (P-P).

Patches of the association developed at higher mountain elevations were observed in extensive complexes of bilberries, and subalpine mat grass communities. Within the mentioned vegetation, the phytocoenoses of Pogonato-Oligotrichetum (Herzog 1943) Balcerk. 1984 have usually been observed directly on the routes.

It is tourism that plays an important role in the origin and persistence of the Nardo-Gnaphalietum phytocoenoses. Most often, it triggers substratum destabilisation on one hand, and treading on the other. The former limits density of the herb layer and generates small eroded places with bare soil. A geobotanical consequence of that is natural seeding and germination of Gnaphalium supinum and the occurrence of orthotropous mosses connected with the freshly eroded areas; i.e. Oligotrichum hercynicum, Pogona-

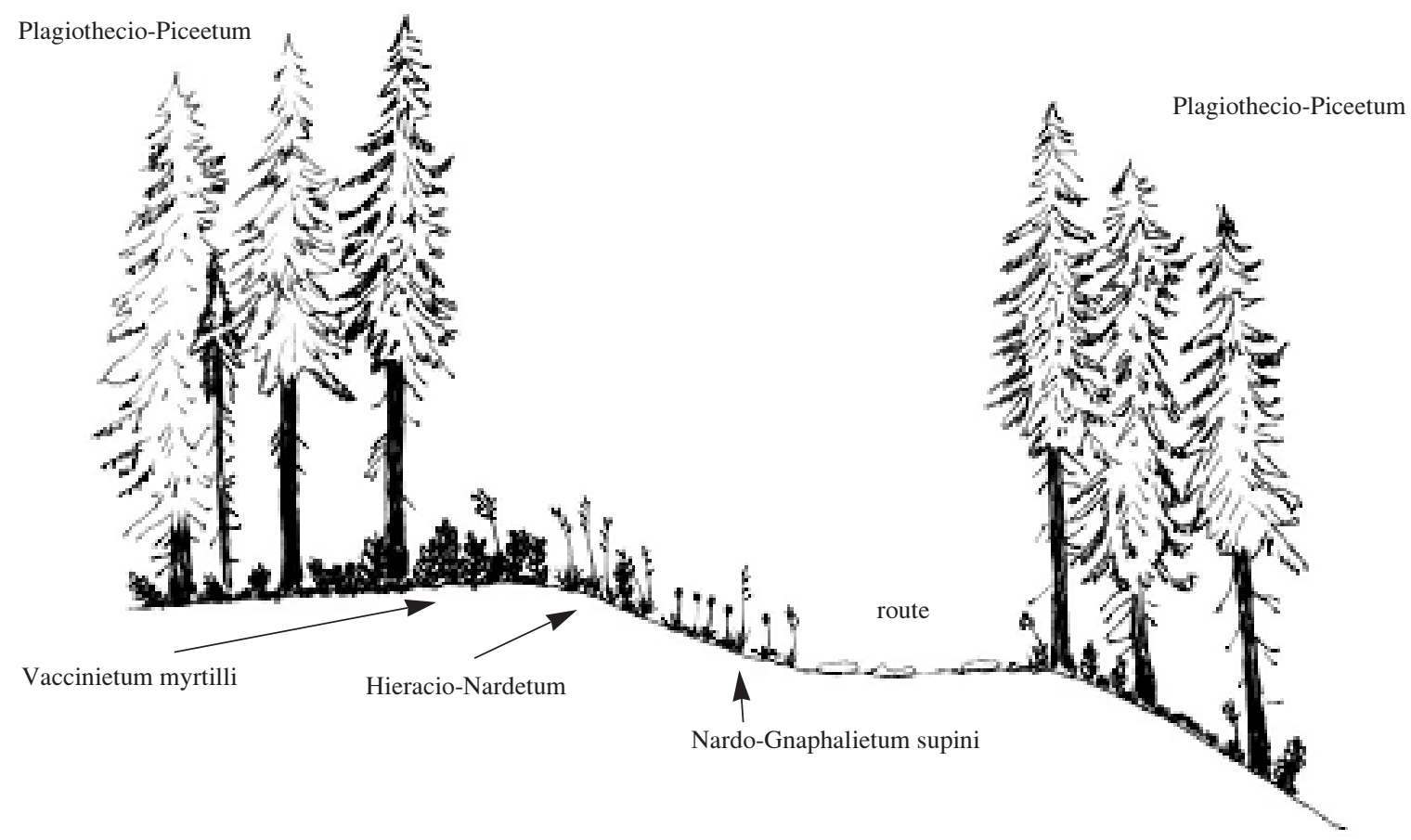

Fig. 1. Spatial pattern of vegetation accompanying the route to Trzydniowiański Wierch (the Western Tatra Mountains). 
TABLE 1. Nardo-Gnaphalietum supini Bartsch et Bartsch ex Müller 1948 from the Tatra Mts. and the Babia Góra Massif.

\begin{tabular}{|c|c|c|c|c|c|c|c|c|c|c|c|c|c|c|c|c|c|c|}
\hline Succesive number of relevé & 1 & 2 & 3 & 4 & 5 & 6 & 7 & 8 & 9 & 10 & 11 & 12 & 13 & 14 & 15 & 16 & & \\
\hline Number of relevé in the field & 39 & 58 & 619 & 49 & 9519 & 9520 & 511 & 507 & 506 & 509 & 59 & 508 & 549 & 396 & 551 & 512 & & \\
\hline day & 3 & 3 & 4 & 8 & 10 & 10 & 7 & 7 & 7 & 7 & 4 & 7 & 10 & 13 & 10 & 7 & & \\
\hline \multirow[t]{2}{*}{ month } & 7 & 8 & 7 & 7 & 8 & 8 & 8 & 8 & 8 & 8 & 8 & 8 & 8 & 9 & 8 & 8 & & $\mathrm{C}$ \\
\hline & 99 & 99 & 97 & 94 & 95 & 95 & 96 & 96 & 96 & 96 & 99 & 96 & 96 & 97 & 96 & 96 & $\mathrm{C}$ & $\mathrm{O}$ \\
\hline Herb layer cover [\%] & 45 & 50 & 60 & 20 & 35 & 35 & 55 & 15 & 40 & 15 & 40 & 30 & 45 & 50 & 35 & 15 & $\mathrm{O}$ & V \\
\hline Moss layer cover [\%] & 80 & 20 & 35 & 20 & 25 & 5 & 10 & 15 & 10 & 30 & 90 & 40 & 85 & 25 & 30 & 60 & $\mathrm{~N}$ & $\mathrm{E}$ \\
\hline Stone cover $[\%]$ & 5 & 35 & 10 & 30 & 5 & 10 & 10 & 10 & 5 & 15 & 5 & 25 & 5 & zn & 20 & 20 & $\mathrm{~S}$ & $\mathrm{R}$ \\
\hline Altitude in [m] & 1490 & 1770 & 1380 & 1150 & 1085 & 1085 & 1475 & 1315 & 1315 & 1400 & 1455 & 1400 & 1355 & 1410 & 1610 & 1490 & $\mathrm{~T}$ & \\
\hline Exposure & NW & - & NEE & $?$ & - & - & $?$ & W & W & NNW & NW & NNW & $\mathrm{NE}$ & NNE & SW & NW & A & $\mathrm{C}$ \\
\hline Inclination [ $\left.{ }^{\circ}\right]$ & 3 & - & 5 & 20 & - & - & 5 & 10 & 5 & 5 & 5 & 5 & 5 & 3 & 30 & 20 & $\mathrm{~N}$ & $\mathrm{O}$ \\
\hline Size of a patch $[\mathrm{cm}]$ & $100 x$ & $200 x$ & - & $87 \times$ & $70 \times$ & $65 x$ & - & $70 \times$ & $125 x$ & - & $350 x$ & $70 x$ & $120 x$ & - & - & - & $\mathrm{C}$ & E \\
\hline Area of relevé $\left[\mathrm{m}^{2}\right]$ & 0.3 & $\begin{array}{c}150 \\
3\end{array}$ & 1.5 & 0.6 & $\begin{array}{l}35 \\
0.2\end{array}$ & $\begin{array}{l}00 \\
0.4\end{array}$ & 0.3 & $\begin{array}{l}40 \\
0.3\end{array}$ & $\begin{array}{l}40 \\
0.5\end{array}$ & 0.5 & $\begin{array}{c}115 \\
4\end{array}$ & $\begin{array}{l}40 \\
0.3\end{array}$ & $\begin{array}{l}30 \\
0.3\end{array}$ & 1 & 0.5 & 0.5 & & \\
\hline Number of species & 18 & 14 & 16 & 14 & 16 & 17 & 12 & 12 & 15 & 14 & 18 & 15 & 16 & 13 & 12 & 16 & & \\
\hline Mountain range & $\mathrm{T}$ & $\mathrm{T}$ & $\mathrm{T}$ & $\mathrm{T}$ & $\mathrm{T}$ & $\mathrm{T}$ & $\mathrm{T}$ & $\mathrm{T}$ & $\mathrm{T}$ & $\mathrm{T}$ & $\mathrm{T}$ & $\mathrm{T}$ & $\mathrm{T}$ & BG & $\mathrm{T}$ & $\mathrm{T}$ & & \\
\hline \multicolumn{19}{|l|}{ I. Ch. Salicetea herbaceae } \\
\hline Gnaphalium supinum & $2 \mathrm{~b} .2$ & $2 \mathrm{~b} .1$ & 3.2 & +.2 & 1.2 & 1.2 & $2 b .3$ & 1.2 & $2 a .2$ & $2 \mathrm{a} .2$ & $2 \mathrm{~b} .2$ & $2 \mathrm{a} .2$ & 2a.2 & 3.2 & $2 a .2$ & $2 \mathrm{a} .2$ & $\mathrm{~V}$ & 1441 \\
\hline Nardia scalaris D & . & . & + & . & + & + & . & . & . & + & . & + & 2.2 & 2.2 & 1.2 & $2 b .3$ & III & 391 \\
\hline Pohlia drummondii & . & . & . & . & . & . & . & . & . & . & . & $\mathrm{r}$ & 2.2 & . & . & . & I & 110 \\
\hline Kiaeria starkei & 1.2 & . & . & $\mathrm{r}$ & . & . & . & . & . & . & . & . & 1.1 & . & . & 1.1 & II & 94 \\
\hline Sedum alpestre & . & $\mathrm{r}$ & . & . & . & . & . & . & . & . & . & . & . & . & 1.1 & 1.2 & & 63 \\
\hline Cerastium cerastoides & . & . & . & . & . & + & . & . & . & . & . & . & . & . & + & +.2 & & 9 \\
\hline \multicolumn{19}{|l|}{ II. Ch. Nardo-Callunetea } \\
\hline Nardus stricta & + & & 2a.1 & +.2 & +.2 & 1.2 & $2 \mathrm{a} .2$ & +.2 & 2a. 2 & 1.2 & + & 1.2 & 2a.2 & $2 \mathrm{~b} .1$ & $\mathrm{r}$ & . & $\mathrm{V}$ & 485 \\
\hline Potentilla aurea & . & $2 \mathrm{a} .1$ & . & +.2 & + & $2 \mathrm{a} .2$ & $\mathrm{r}$ & $\mathrm{r}$ & . & . & + & . & $\mathrm{r}$ & . & . & + & III & 139 \\
\hline Carex leporina & . & . & . & + & $(+.2)$ & + & . & . & . & . & . & . & . & . & . & . & & 38 \\
\hline Luzula multiflora & . & . & + & $\mathrm{r}$ & . & . & . & . & . & . & . & . & . & . & . & . & & 4 \\
\hline Carex pilulifera & . & . & + & . & . & . & . & . & $\mathrm{r}$ & . & . & . & . & . & . & . & & 4 \\
\hline Potentilla erecta & . & . & . & . & + & . & . & . & . & . & . & . & . & . & . & . & & 3 \\
\hline Gnaphalium norvegicum & . & . & . & . & . & . & . & . & . & . & 1.2 & . & . & . & . & . & & 31 \\
\hline \multicolumn{19}{|l|}{ III. Ch. Pogonato-Dicranelletea } \\
\hline Oligotrichum hercynicum & + & 1.2 & 2.3 & 2.3 & $2 \mathrm{a} .3$ & + & + & + & + & + & 3.2 & +.2 & + & 1.2 & 1.2 & & $\mathrm{~V}$ & 634 \\
\hline Ditrichum heteromallum & . & . & . & . & 1.1 & + & + & 1.3 & + & . & $2 \mathrm{~b} .2$ & . & . & . & 1.2 & . & III & 228 \\
\hline Pogonatum urnigerum & + & . & . & 1.1 & . & + & . & . & $\mathrm{r}$ & 1.2 & 2.2 & +.2 & + & $\mathrm{r}$ & . & + & IV & 189 \\
\hline Dicranella heteromalla & . & 1.2 & + & . & . & . & . & . & 1.2 & $\cdot$ & $\cdot$ & $\mathrm{r}$ & . & + & . & $\cdot$ & II & 69 \\
\hline \multicolumn{19}{|l|}{ IV. Others } \\
\hline Deschampsia flexиosa & $\mathrm{r}$ & . & 1.2 & . & +.2 & . & 1.2 & 1.2 & 1.2 & + & 1.1 & 1.2 & 1.2 & + & 1.2 & $\mathrm{r}$ & $\mathrm{V}$ & 261 \\
\hline Picea abies juv. & . & . & $\mathrm{r}$ & + & $\mathrm{r}$ & + & . & $\mathrm{r}$ & $\mathrm{r}$ & . & $\mathrm{r}$ & . & . & . & . & . & III & 9 \\
\hline Anthoxanthum alpinum & + & . & +.2 & . & . & . & 1.2 & . & . & +.2 & . & + & + & . & + & 1.2 & III & 81 \\
\hline Vaccinium myrtillus & + & . & + & . & . & . & $\mathrm{r}$ & $\mathrm{r}$ & . & $\mathrm{r}$ & + & . & . & . & $\mathrm{r}$ & . & III & 12 \\
\hline Mutellina purpurea & + & + & . & . & . & . & . & . & . & . & . & . & $\mathrm{r}$ & . & . & $\mathrm{r}$ & II & 8 \\
\hline Agrostis rupestris & 1.3 & $2 \mathrm{~b} .1$ & 1.1 & . & . & . & $\mathrm{r}$ & . & . & . & . & . & . & . & + & . & II & 191 \\
\hline Agrostis capillaris & . & . & . & 2.2 & $2 \mathrm{~b} .3$ & + & . & . & + & . & . & . & . & . & . & . & II & 241 \\
\hline Deschampsia caespitosa & $\mathrm{r}$ & + & . & . & $2 \mathrm{a} .2$ & $2 a .2$ & . & . & . & . & + & . & . & . & . & $\mathrm{r}$ & II & 133 \\
\hline Polytrichastrum pallidisetum & . & . & . & . & 1.2 & 1.2 & $2 \mathrm{a} .2$ & & . & . & . & . & . & . & . & . & I & 125 \\
\hline Lophozia sudetica & . & . & + & . & . & . & + & . & . & . & $2 \mathrm{~b} .2$ & + & . & . & 1.2 & + & II & 169 \\
\hline Cephalozia bicuspidata & . & . & . & . & . & . & . & . & . & . & 3.2 & $\mathrm{r}$ & 2.2 & . & . & . & I & 344 \\
\hline Homogyne alpina & . & . & . & . & . & . & . & . & . & + & . & +.2 & $2 \mathrm{a} .3$ & . & . & . & & 69 \\
\hline Pleurozium schreberi & . & . & . & . & . & . & . & & . & $\mathrm{r}$ & . & + & $\mathrm{r}$ & . & . & . & & 4 \\
\hline Rumex acetosella & . & . & . & + & . & + & . & . & . & . & . & . & . & . & . & 1.2 & & 38 \\
\hline Luzula luzuloides & . & . & . & . & . & . & . & + & + & . & . & . & . & . & . & $\mathrm{r}$ & & 7 \\
\hline Rubus idaeus & . & . & . & . & . & . & . & $\mathrm{r}$ & +.2 & . & . & . & . & $\mathrm{r}$ & . & . & I & 4 \\
\hline Jungermannia sphaerocarpa & 4.3 & . & . & . & . & . & . & . & $\cdot$ & . & . & . & . & . & . & . & I & 391 \\
\hline Campanula polymorpha & 2.3 & . & + & . & . & . & . & & . & . & . & . & . & . & . & . & I & 113 \\
\hline
\end{tabular}

Sporadic species: I. Luzula alpino-pilosa 3(+); Marsupella brevissima 10(+); Sibbaldia procumbens 2(+). II. Geum montanum 2(r). III. Alchemilla sp. 5(r); Anastrophyllum minutum 12(r); Bryum cfr. bicolor 2 (2.2); Calamagrostis villosa 14(+); Festuca airoides 1(r); Gnaphalium sylvaticum 6(r); Hieracium alpinum 13(r); H. atratum 8(r), 9(r); H. murorum 11 (1.1); Leontodon autumnalis 4(r); Lophozia cfr. sudetica 10(+); L. wenzelii 10(+); Luzula sylvatica 11(r); Oxalis acetosella 6(r); Phleum commutatum 2(+), 11(r); Poa alpina 2(+); Pohlia nutans 7(r); P. obtusifolia 4(r); Polytrichastrum alpinum 8(+), 9(r); P. formosum 1(+), 14 (+.2); Polytrichum piliferum 3 (2.1); Racomitrium elongatum 12 (2a.2), 16 (1.2); R. ericoides 1(+), 10 (1.2); Rhytidiadelphus squarrosus 1(+), 4(r); Salix silesiaca 14(+); Sanionia uncinata 1(+); Scapania curta 5(+), 9(r); Senecio nemorensis 11(r); S. subalpinus 11(r); Soldanella carpatica 16 (+.2); Taraxacum sp. 2(r), 5(r), 6(r); Vaccinium vitis-idaea 14(+).

Key: BG - the Babia Góra Massif, T - the Tatra Mountains. 
TABLE 2. Differentiation of the floristic composition of Nardo-Gnaphalietum supini Bartsch et Bartsch ex Müller 1948 in Europe.

Number of column

Number of relevés

Total number of species in the table (relevé)

Average number of species in relevé

Minimal number of species in relevé

Maximal number of species in relevé

Altitude in [m]

1
16
72

$72 \quad 42$

$12 \quad 15$

$12 \quad 11$

12

$\begin{array}{cccc}18 & 18 & 32 & 27 \\ 1085-1770 & 1660-1820 & 1920-2020 & 1790-2030\end{array}$

59

20

13

5

(11)

(10)

(12)

(14)

(11)

10

1980

$1900 \quad 1420$

\begin{tabular}{|c|c|c|c|c|c|c|c|c|c|c|}
\hline Mountain range & \multicolumn{3}{|c|}{ Carpathians } & \multicolumn{4}{|c|}{ Alps } & \multicolumn{3}{|c|}{ Black Forest } \\
\hline \multicolumn{11}{|l|}{ Snow-bed species } \\
\hline Gnaphalium supinum & V 1441 & V 3875 & V 50 & IV 130 & 2.4 & 3.4 & 2.3 & 2.3 & + & + \\
\hline Veronica alpina & & I 6 & I 6 & II 20 & + & + & & + & & \\
\hline Kiaeria starkei & II 94 & I 219 & & III 860 & & & 1.2 & & & 1 \\
\hline Sedum alpestre & I 63 & IV 31 & I 6 & & & & & & & \\
\hline Nardia scalaris & III 391 & & & & & & & & & 1 \\
\hline Ranunculus crenatus & & IV 144 & & & & & & & & \\
\hline Soldanella hungarica & & IV 31 & & & & & & & & \\
\hline Anthelia juratzkana & & III 250 & & & & & & & & \\
\hline Plantago gentianoides & & & II 19 & & & & & & & \\
\hline Ranunculus pseudomontanus & & & II 19 & & & & & & & \\
\hline Tanacetum alpinum & & & II 13 & & & & & & & \\
\hline Plantago atrata & & & II 19 & I 100 & & 1.2 & + & & & \\
\hline Plantago alpina & & & & IV 2600 & & & & +.2 & & \\
\hline Salix herbacea & & & & III 30 & & & & & & \\
\hline Sagina saginoides & & & & II 20 & & & & & + & \\
\hline \multicolumn{11}{|l|}{ Mat grassland species } \\
\hline Nardus stricta & V 485 & V 1725 & V 3563 & V 2860 & + & 1.2 & + & 4.4 & 4 & 4 \\
\hline Potentilla aurea & III 139 & V 100 & V 856 & I 10 & + & & 1.2 & 2.2 & 3 & \\
\hline Geum montanum & I 1 & V 313 & V 100 & & & & & & & \\
\hline Carex leporina & II 38 & I 6 & & & & & & & & \\
\hline Carex pilulifera & I 4 & & & & & & & & & \\
\hline Potentilla erecta & I 3 & & & & & & & & & \\
\hline Festuca rubra & & & II 13 & & & & & & & \\
\hline Leucorchis albida & & & IV 31 & & & & & & & \\
\hline
\end{tabular}

Species attesting to great dynamics of substrate Oligotrichum hercynicum

Ditrichum heteromallum

Pogonatum urnigerum

Lophozia sudetica

Dicranella heteromalla

V 634
III 228
III 189
III 169
II 69

\section{Others}

Mutellina purpurea

Polytrichastrum alpinum

Agrostis rupestris

Anthoxanthum alpinum

Deschampsia caespitosa

Homogyne alpina

Poa alpina

Deschampsia flexuosa

Festuca airoides

Campanula abietina

Campanula alpina

Taraxacum alpinum

Cetraria islandica

Vaccinium myrtillus

Picea abies

Agrostis capillaris

Poa media

Avenula versicolor

Poa annua

Phleum commutatum

Phyteuma nanum

Rhododendron myrtifolium

Leontodon helveticus

Racomitrium canescens

\begin{tabular}{|c|c|c|c|c|c|c|c|c|c|}
\hline II 8 & IV 981 & V 525 & V 320 & 2.2 & 2.3 & 3.3 & +.2 & 4 & 4 \\
\hline I 4 & V 794 & & & 1.2 & 1.2 & + & & & 1 \\
\hline II 191 & II 125 & II 69 & & & & & & & \\
\hline III 81 & IV 263 & I 63 & II 20 & & & & & & \\
\hline II 133 & IV 38 & II 13 & I 10 & & & & & & \\
\hline I 69 & II 13 & III 238 & IV 40 & & & & & & \\
\hline I 3 & II 13 & II 13 & II 110 & & + & + & & & \\
\hline V 261 & I 6 & I 6 & II 20 & & & & 1.2 & & \\
\hline \multirow[t]{5}{*}{ I 1} & II 69 & I 6 & & & & & & & \\
\hline & I 6 & IV 31 & & & & & & & \\
\hline & II 13 & II 13 & & & & & & & \\
\hline & II 13 & II 13 & & & & & & & \\
\hline & I 63 & II 13 & I 10 & & & & & & \\
\hline $\begin{array}{c}\text { III } 12 \\
\text { III } 9\end{array}$ & II 13 & & & & & & & & \\
\hline \multirow[t]{4}{*}{ II 241} & & & I 10 & & & & & & \\
\hline & & V 3569 & & & & & & & \\
\hline & & V 256 & I 10 & & & & + & & \\
\hline & & III 25 & I 10 & & & & & & \\
\hline \multirow[t]{5}{*}{ I 4} & & III 25 & & & & & & & \\
\hline & & III 25 & & & & & & & \\
\hline & IV 38 & & & & & & & & \\
\hline & & & III 210 & 1.2 & & 2.2 & +.2 & + & 1 \\
\hline & & & & 2.3 & & 1.2 & & & \\
\hline
\end{tabular}


tum urnigerum, Dicranella heteromalla and Ditrichum heteromallum. All these taxa are the components of PogonatoOligotrichetum, a common association in this area. It seems that the presence of snow-bed plants in patches of Nardo-Gnaphalietum is determined more by treading of the soil by tourists rather, than by longer coverage of snow. The latter may be of higher importance in the montane zones.

Identification of Nardo-Gnaphalietum phytocoenoses in the field does not provide any substantial problems. Gnaphalium supinum and Nardus stricta are structural dominants in the communities' coverage. The floristic composition of the association includes additionally an admixture of the species characteristic of the classes: Nardo-Callunetea Prsg. 1949 (most often Potentilla aurea, which is linked to trodden places in anthropogenic habitats), Salicetea herbaceae Br.-B1. 1948 (mainly mosses), and Pogonato-Dicranelletea v. Hübschmann 1975 (components of PogonatoOligotrichetum). Forms of the community known from the higher elevations are characterised by a share of taxa typical to alpine grasslands of Caricetea curvulae Br.-B1. 1948, such as Agrostis rupestris, which prefers places with unstable substratum.

In the Tatra Mountains, Nardo-Gnaphalietum supini is a common plant community due to its anthropo-auksochoric character. Patches of the association were also found on the Babia Góra Massif; from where they were documented with one relevé (Table 1, rel. 14). The secondary character of the patches of Nardo-Gnaphalietum supini has been previously postulated by Oberdorfer (1957).

\section{DISTRIBUTION OF THE STUDIED PATCHES}

The Babia Góra Massif: Izdepczyska (Table 1, rel. 14). The Tatra Mts: At the route to Trzydniowiański Wierch
Mt. (from Dolina Jarząbcza Valley) near Wielki Przysłop (Table 1, rel. 1); Przełęcz Wyżnia Kondracka Pass near a tourist trail (2); Dolina Wyżnia Chochołowska Valley, near a tourist trail $(3,4)$; above the crossroads of the routes to Przełęcz Iwaniacka and to Dolina Starorobociańska (5, 6); Dolina Jarząbcza, at the route to Trzydniowiański Wierch above the stream flowing down from Szeroki Żleb $(7,16)$; Dolina Jarząbcza, at the route to Trzydniowiański Wierch above Potok Jarząbczy $(8,9)$; Dolina Jarząbcza, at the route to Trzydniowiański Wierch above Jarząbcze Szałasiska $(10,12)$; slightly below Przełęcz Iwaniacka on the side of Dolina Chochołowska (11); Dolina Starorobociańska, above Starorobociańska Rówień near a tourist trail (13); Dolina Starorobociańska, near the tourist trail (15).

Elevations above sea level of the studied patches are given in the head of Table 1.

\section{DISCUSSION}

A synthetic comparison of the floristic composition of Nardo-Gnaphalietum supini from the Alps, the Black Forest, and both Western and Eastern Carpathians (Table 2), shows that this syntaxon has a unique floristic combination among all snow-beds communities. It is distinguished by a representation of the species of the class Nardo-Callunetea. It is not possible, however, to point out characteristic taxa for the association. Phytocoenoses known from the Carpathians are floristically richer than those described from the Alps and the Black Forest. The highest number of taxa were recorded in patches of the association from the Tatra Mountains and the Babia Gora Massif. This is the consequence of two facts: Firstly, the documentation from the Western Carpathians comprises the highest number of relevés; secondly, they encompass the highest difference of altitude, i.e. 1085 to $1770 \mathrm{~m}$. All the other compared phy-

Sporadic species: Agrostis agrostiflora 4 (I 750); A. alpina 3 (I 6), 4 (I 10); Alchemilla decumbens 4 (I 100); A. monticola 4 (I 10); A. obtusa 2 (I 6); A. sp. 1 (I 1); A. vulgaris 3 (I 6); Anastrophyllum minutum 1 (I 1); Androsace chamaejasme 4 (I 10); Antennaria dioica 3 (I 6); Arabis alpina 2 (I 6); Barbilophozia floerkei 10(+); B. lycopodioides 4 (I 10); Bartsia alpina 4 (I 10); Bryum cfr. bicolor 1 (I 109); B. sp. 4 (II 760); Calamagrostis villosa 1 (I 3); Campanula polymorpha 1 (I 113); C. scheuchzeri 4 (I 10); Cardaminopsis arenosa ssp. borbasii 2 (I 6); Carex atrata 3 (I 6); C. capillaris 4 (I 10); C. nigra 4 (I 10); Cephalozia bicuspidata 1 (I 344); Cerastium alpinum 3 (II 19); C. caespitosum 3 (I 6); Cladonia sp. 4 (II 20); Cratoneuron filicinum 4 (I 1250); Distichum capillaceum 4 (II 20); Euphrasia minima 4 (II 20); Festuca nigrescens 2 (I 6); F. picta 3 (II 13); F. rupicaprina 4 (II 20); Galium anisophyllon 4 (I 10); Gentiana bavarica 4 (II 20); G. kochiana 8(r); G. nivalis 4 (I 10); G. punctata 4 (I 10), 8(+); G. verna 2 (I 6); Gnaphalium norvegicum 1 (I 31 ), 2 (I 6); G. sylvaticum 1 (I 1); Hieracium alpinum 1 (I 1); H. atratum 1 (I 1), 3 (II 13); H. lachenalii 1 (I 1); H. murorum 1 (I 31); Huperzia selago 2 (I 6); Hypericum grisebachii 2 (I 6); Juncus trifidus 2 (II 13); Jungermannia gracillima 10(+); J. sphaerocarpa 1 (I 391); Leontodon autumnalis 1 (I 1); L. hispidus 4 (I 10); Lophozia wenzelii 1 (I 3), 9(+); Luzula campestris 4 (I 10); L. luzuloides 1 (I 7); L. multiflora 1 (I 4), 8(+); L. spicata 3 (II 13); L. sylvatica 1 (I 1); Marsupella brevissima 1 (I 3); Meum athamanticum 9(+), 10 (1); Nardia geoscyphus 4 (I 10); Oxalis acetosella 1 (I 1); Phleum raeticum 4 (II 20); Pleurozium schreberi 1 (I 4); Poa minor 4 (I 10); P. supina 2 (I 6); Pohlia drummondii 1 (I 110), 10 (1); P. nutans 1 (I 1); P. obtusifolia 1 (I 1); Polygonum viviparum 4 (I 10), 5 (1.1), 6(+); Polytrichastrum formosum 1 (I 6), 4 (II 110); P. pallidisetum 1 (I 125); P. sexangulare 4 (I 10); Polytrichum juniperinum 4 (II 450); P. piliferum 1 (I 109); P. sp. 9(r); Potentilla brauniana 4 (I 100); Primula minima 2 (I 6), 3 (I 6); Racomitrium elongatum 1 (I 94); R. ericoides 1 (I 34); R. lanuginosum 2 (I 6); R. sp. 7(r), 8(+); Ranunculus alpestris 4 (I 10); Rhododendron kotschyi 3 (I 6); Rhytidiadelphus squarrosus 1 (I 4); Rubus idaeus 1 (I 4); Rumex acetosella 1 (I 38), 8(r); Salix retusa 4 (I 10); S. silesiaca 1 (I 3); Sanionia uncinata 1 (I 3), 4 (II 110); Scapania curta 1 (I 4); Senecio nemorensis 1 (I 1); S. subalpinus 1 (I 1); Selaginella selaginoides 4 (I 10); Sesleria albicans 4 (I 10); Sibbaldia procumbens 1 (I 3 ), 4 (I 100); Soldanella carpatica 1 (I 3); Taraxacum sp. 1 (I 2); Thymus alpestris 2 (I 6); T. montanus 3 (II 13); Trifolium repens 3 (II 13), 4 (I 10); Vaccinium gaultherioides 3 (I 6); V. vitis-idaea 1 (I 3), 3 (I 6); Veratrum album 2 (I 6); Veronica bellidioides 3 (I 6); V. officinalis 3 (II 13 ).

Explanations of data included in the columns: 1 - GÓRSKI (orig.), the Western Carpathians; 2 - COLDEA (1990, Table 23), the Eastern Carpathians, the Rodney Mountains; 3 - RESMERITA (1975, Table 2, rel. 1,2 and Table 3, rel. 1, 2, 5, 6, 8, 9), the Eastern Carpathians, Retezat; 4 - EGGENSBERGER (1994, Table 15, rel. 39-43), the Eastern Alps, the Ammergauer Alpen; 5, 6, 7 - THIMM (1953, synthetic table 25, columns 4, 5, 6), the Tyrol Alps, Rofan; 8 - OBERDORFER (1950, Table 16, rel. 1), the Alps, Allgaeu; 9 - BARTSCH J., BARTSCH M. (1940, page 113), first ever made relevé of Nardo-Gnaphalietum, the Black Forest, Feldberg; 10 - MÜLLER K. (1948, page 314-315), second ever made relevé of Nardo-Gnaphalietum, the Black Forest, Feldberg.

Measurements of species share presented in columns: 1, 2, 3, 4- cover coefficient and phytosociological constancy in classes; 5, 6, 7, 8 - species' quantity and sociability; 9, 10 - species' quantity. 


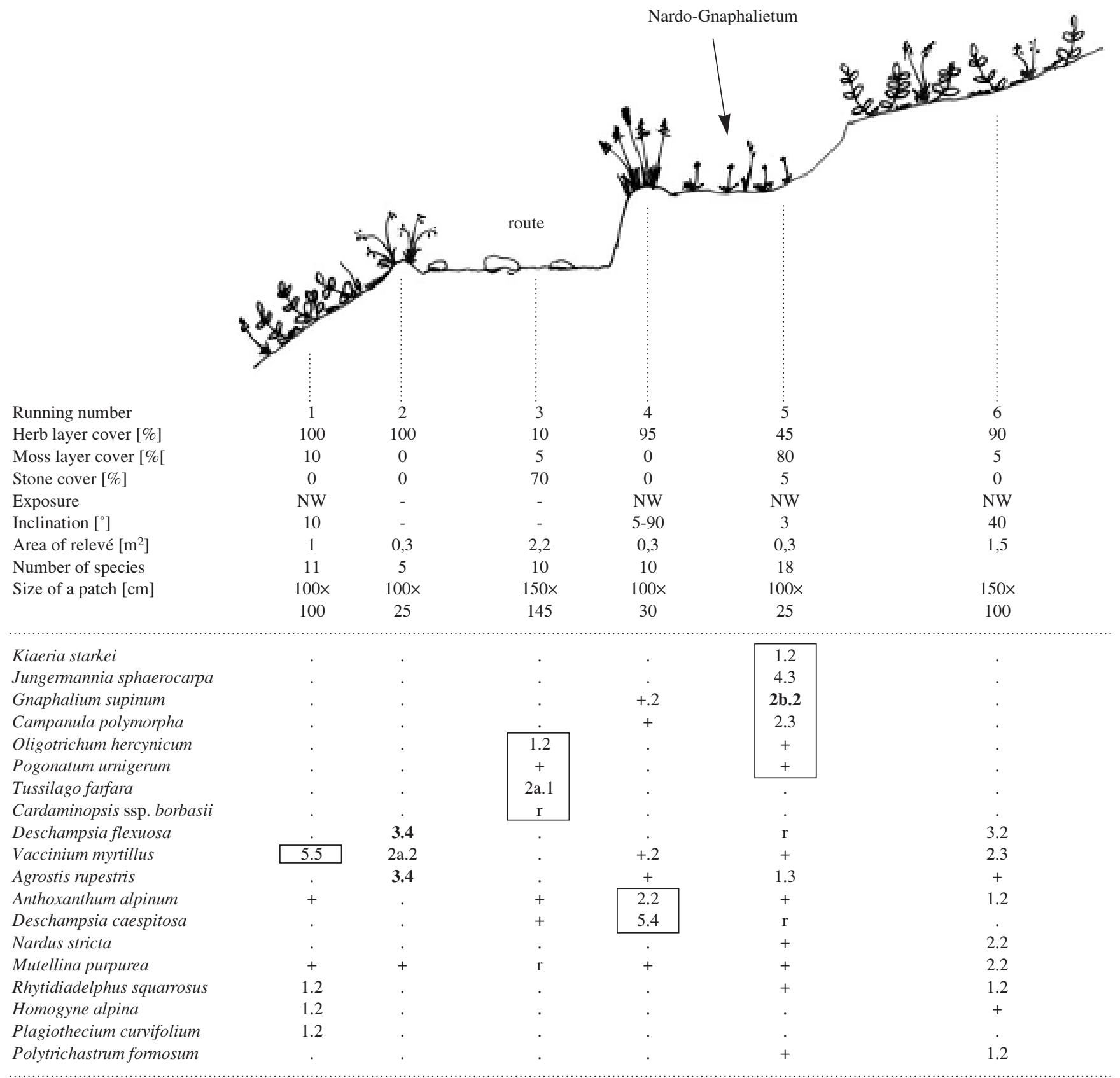

Aconitum firmum 4(r); Epilobium alsinifolium 3(+); Festuca airoides 4(+), 5(r); Festuca picta 2(+), 6(+); Geum montanum 1(+); Hieracium sp. 3(r); Hylocomium splendens $1(+)$; Oxalis acetosella 6(r); Pleurozium schreberi 1(+); Potentilla aurea 1(+), 6(+); Racomitrium ericoides 5(+); Sanionia uncinata 5(+); Soldanella carpatica 1(+), $4(+.2), 6(+)$; Taraxacum sp. 3(r).

Fig. 2. Phytocoenoses of Nardo-Gnaphalietum supini in a complex with the subalpine bilberry (Vaccinium myrtillus) community developing on additional footpath above the tourist route in the Tatra Mountains [at the route to Trzydniowiański Wierch (from Dolina Jarząbcza) near Wielki Przysłop, $1490 \mathrm{~m}$ a.s.1.]. rel. 1 - Vaccinietum myrtilli; 2 - a community with Agrostis rupestris developing on the lower (descending) side of a slope; 3 - fragmentally developed Pogonato-Oligotrichetum and a natural seeding of Tussilago farfara observed between the stabilised rocky slabs of a mountain route; 4 - a community with Deschampsia caespitosa; 5 - Nardo-Gnaphalietum supini above the main tourist track, on additional, rarely used footpath; 6 - alpine grassland Hieracio alpini-Nardetum being overgrown by bilberry and wavy hair-grass.

tosociological data refer exclusively to the high mountain zones.

Englisch (1993) has postulated a need of revision of snow-bed communities with Nardus stricta. Moreover, he formulated a hypothesis that mat-grass communities from the Alps and average mountains of Germany could be forms of two different associations. The presented synthetic material (Table 2) was still insufficient to distinguish two separate units. However, the compared data were qu- antitatively poor. Therefore, it is possible that enriching of the documentation by additional relevés would allow to show clear floristic differences between the analysed snowbed vegetation. However, it may be difficult because of the lack of characteristic species of Nardo-Gnaphalietum supini. In this context, the discussed unit remains the central association (according to Dierschke 1981, 1994) within the alliance Nardo-Salicion herbaceae distinguished by Englisch (1999). 
A constant share of the group of moss species growing on the freshly eroded soil manifests the distinctiveness of the patches recorded from the Western Carpathians. Such phytocoenoses have a specific floristic composition and are easily distinguishable in relation to local habitats' variability. This enables to give them the status of a separate subassociation, namely Nardo-Gnaphalietum supini oligotrichetosum hercynici subass. nova [Typus: Table 1, rel. 3]. The relevé made by Müller (1948) in the Black Forest should also be regarded as representing the described unit (cf. Table 2 , col. 10 in this article).

\section{ACKNOWLEDGEMENTS}

This study was supported by the State Committee for Scientific Research, Warsaw, Poland (grants nos 6 PO4G 03518 and 6PO4G 027 21).

\section{LITERATURE CITED}

BARKMAN J.J., DOING H., SEGAL S. 1964. Kritische Bemerkungen und Vorschläge zur quantitativen Vegetationsanalyse. Acta Bot. Neerl. 13: 394-419.

BARTSCH J., BARTSCH M. 1940. Vegetationskunde des Schwarzwaldes. Pflanzensoziologie 4: 1-229.

BOLLETER R. 1921. Vegetationsstudien aus dem Weisstannental. Jahrb. St. Gall. Naturwiss. Ges. 57 (2): 1-121.

COLDEA G.H. 1990. Munti Rodnei. Studiu geobotanic. Ed. Acad., Bucuresti, pp. 183.

DIERSCHKE H. 1981. Zur syntaxonomischen Bewertung schwach gekennzeichneter Pflanzengesellschaften. In: Berichte der Internationalen Symposien der Internationalen Vereinigung für Vegetationskunde. Syntaxonomie. Rinteln 31.3.-3.4.1980. Dierschke H. (ed.). Vaduz, pp. 109-122.

DIERSCHKE H. 1994. Pflanzensoziologie. Grundlagen und Methoden. Verlag Eugen Ulmer, Stuttgart, pp. 683.
EGGENSBERGER P. 1994. Die Pflanzengesellschaften der subalpinen und alpinen Stufe der Ammergauer Alpen und ihre Stellung in den Ostalpen. Ber. Bayer. Bot. Ges. 8: 1-239.

ENGLISCH T. 1993. Salicetea herbaceae. In: Die Pflanzengesellschaften Österreichs. Teil 2. Natürliche waldfreie Vegetation. Grabherr G., Mucina L. (eds). Gustav Fischer Verlag, Jena, pp. 382-401.

ENGLISCH T. 1999. Multivariante Analysen zur Synsystematik und Standortsökologie der Schneebodenvegetation (Arabidetalia caeruleae) in den Nördlichen Kalkalpen. Stapfia 59: 1-211.

GROLLE R. 1983. Hepatics of Europe including the Azores: An annotated list of species, with synonyms from the recent literature. Journ. Bryol. 12 (3): 403-459.

MIREK Z., PIĘKOŚ-MIRKOWA H., ZAJĄC A., ZAJĄC M. 1995. Vascular plants of Poland: a checklist. Polish Bot. Stud. Guideb. Ser. 15: 1-303.

MÜLLER K. 1948. Die Vegetationsverhältnisse im Feldberggebiet. In: Der Feldberg im Schwarzwald. Naturwissenschaftliche, landwirtschaftliche, forstwirtschaftliche, geschichtliche und siedlungsgeschichtliche Studien. Müller K. (ed.). L. Bielefelds Verlag KG, Freiburg, pp. 211-362.

OBERDORFER E. 1950. Beitrag zur Vegetationskunde des Allgäu. Beitr. naturk. Forsch. Südwest-Deutschland 9: 29-98.

OBERDORFER E. 1957. Süddeutsche Pflanzengesellschaften. Pflanzensoziologie 10: 1-551.

OCHYRA R., SZMAJDA P., BEDNAREK-OCHYRA H. 1992. List of mosses to be published in atmos. In: Atlas of the geographical distribution of mosses in Poland 8. Ochyra R., Szmajda P. (eds). W. Szafer Institute of Botany, Polish Academy of Sciences, Kraków, Poznań, pp. 9-14.

PAWŁOWSKI B. 1966. Composition and structure of plant communities and methods of their study. In: The vegetation of Poland. W. Szafer (ed.). Pergamon Press, Oxford, London, Edinburgh, New York, Paris, Frankfurt \& PWN-Polish Scientific Publishers, Warszawa, pp. 241-281.

RESMERITA I. 1975. La classe Nardo-Callunetea Prsg. 1949 dans les Carpathes Roumains. Doc. Phytosoc. 9-14: 265-278.

THIMM I. 1953. Die Vegetation des Sonnwendgebirges (Rofan) in Tirol (subalpine und alpine Stufe). Ber. Naturwiss. Med. Ver. Innsbruck 50: 5-166. 